\title{
Pediatric Laryngeal Malignancies: Current Management Protocols and Review of Literature
}

\author{
Sudhir M Naik, A Nanjundappa, H Srihariprasad, Rajshekar Halkud, Purshottam Chavan, Sumit Gupta
}

\begin{abstract}
Background: Malignant lesions of the larynx are very rare in pediatric population as it accounts for less than $0.1 \%$ of all head and neck malignancies in childhood. Apart from human papilloma virus infection, adult risk factors like laryngeal papilloma, gastroesophageal reflux, human immunodeficiency virus (HIV) infection, immunosuppressive therapy, smoking, alcohol abuse, poor oral hygiene and a family history of cancer also play a role in pediatric laryngeal cancers.
\end{abstract}

Case report A 14-year-old boy presented to us with hoarseness of voice since 8 months. He was treated for laryngitis and subacute bacterial laryngitis and later referred to us. He complained of mild dysphagia and the hoarseness of voice. An ulceroproliferative lesion in the left arytenoids and aryepiglottic fold was seen with retroarytenoid extension in the left arytenoids and the left hemilarynx was fixed. The left level II node was enlarged $2 \times 2 \mathrm{~cm}$ and a staged as carcinoma supraglottis T3/ $4 N 1 M x$. The node aspiration biopsy revealed metastatic squamous cell carcinoma.

Intervention: The case was posted for wide field laryngectomy, under ASA grade II. The direct laryngoscopic examination revealed the extent of the tumor, and the frozen sections of biopsy revealed squamous cell carcinoma of the larynx. The surgery was done with the classical Gluck Sorenson incision and the patient was given postoperative radiotherapy.

Conclusion: Pediatric laryngeal cancers are very rare and studies about the etiology, biology of the tumor, diagnostic and management protocols are not standardized. Formulating a protocol for childhood laryngeal cancers management which includes early diagnosis and accurate treatment is essential.

Keywords: Squamous cell carcinoma, Hoarseness, Total laryngectomy, External beam radiation, Human papilloma virus.

Howto cite this article: Naik SM, Nanjundappa A, S rihariprasad H, Halkud R, Chavan P, Gupta S. Pediatric Laryngeal Malignancies: Current Management Protocols and Review of Literature. IntJ Phonosurg Laryngol 2012;2(2):62-65.

\section{Source of support $\mathrm{Nil}$}

Conflict of interest: None declared

\section{INTRODUCTION}

Malignant lesions of the larynx are very rare in pediatric population as it accounts for less than $0.1 \%$ of all head and neck malignancies in childhood. ${ }^{1,2}$ Rehn in 1868 first reported a case of laryngeal cancers in a 3-year-old child. ${ }^{3}$ Extensive literature search revealed that overall 91 cases of laryngeal cancers have been reported with 28 cases below 10 years and 63 cases bel ow 15 years. ${ }^{1,4} \mathrm{M}$ ale predominance is not seen as in adults. ${ }^{5}$
Zahidul et al reported literature research of 98 cases of carcinoma of the larynx in patients less than 20 years of age, which have about 20 cases in the age group less than 12 years. ${ }^{2}$ So a remote differential diagnosis should be in mind as laryngeal cancers in pediatric population accounts for less than $0.1 \%$ of all head and neck malignancies. ${ }^{6-8}$ Incidence in female are nearly equal to male in pediatric laryngeal cancer (female $40 \%$ in pediatric, $10 \%$ in adult cancer). ${ }^{3}$ Risk factor include previous irradiation of papilloma, papillomatosis, malnutrition and human papilloma virus (HPV) infection. ${ }^{3}$

Immunologic and genetic factor which drastically modify the common risk factors, such as tobacco use, previous radiation and chemical carcinogens into definite etiologies also play a role in pediatric patients. ${ }^{9,10}$ J uvenile papillomatosis show malignant degeneration after irradiation and so irradiation as treatment is abandoned. ${ }^{2}$ A dult risk factors like laryngeal papilloma, gastroesophageal reflux, human immunodeficiency virus (HIV) infection, immunosuppressive therapy, exposure to drug use during pregnancy, both active and passive smoking, exposure to certain chemicals (e.g. asbestos), alcohol use, poor oral hygiene and a family history of cancer also play a role in pediatric laryngeal cancers. ${ }^{11}$ Long history of progressive airway obstruction, dysphagia or dysphonia are seen in children but lesions are only diagnosed by a laryngologist by routine mirror or fiber-optic airway examination. ${ }^{3}$ The scarcity of cases, attempt to preserve anatomy and function of Iarynx and for avoidance of complications impedes establishment of treatment protocols in children. ${ }^{3}$ The rarity of case has made treatment protocols difficult while workup is similar to that in adults. ${ }^{12,13} \mathrm{No}$ literature on organ preservation strategies, prognosis, survival rates are reported. ${ }^{3}$

We present a case of squamous cell carcinoma (SCC) in a 15-year-old male, presenting in the advanced stage with laryngeal function compromised and underwent wide field laryngectomy with postoperative concurrent chemoradiotherapy.

\section{CASE REPORT}

A 14-year-old boy presented to us with hoarseness of voice since 8 months. He was treated at various hospitals for laryngitis and subacute bacterial laryngitis and later referred to us for further evaluation. He also complained of mild 


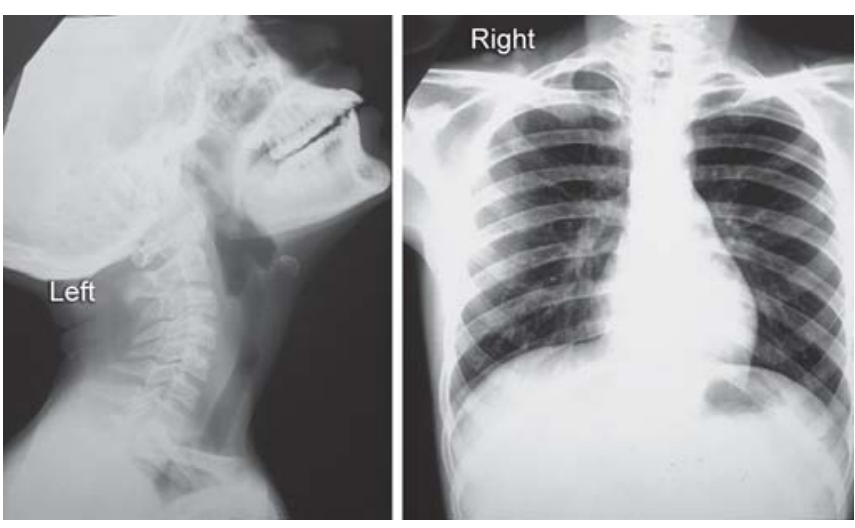

Fig. 1: Chest X-ray being normal and lateral X-ray of the neck showing no subglottic extension

dysphagia and the hoarseness of voice was slowly progressive in type. On indirect laryngoscopic examination revealed an ulceroproliferative lesion in the left arytenoids and aryepiglottic fold. There was a retroarytenoid extension in the left arytenoids and the left hemilarynx was fixed. The left level II node was enlarged $2 \times 2 \mathrm{~cm}$ and the lesion was staged as carcinoma supraglottis T3/4N 1M x (Fig. 1).

The fine needle aspiration biopsy revealed metastatic SCC. With the clinical lesion with metastasis to the neck, staged with computed tomographic (CT) scan, the patient was posted for wide field laryngectomy. The patient was posted for total laryngectomy, as the larynx was functionally compromised and cartilage infiltration seen. The case was posted for wide field laryngectomy, under ASA grade II. The direct laryngoscopic examination revealed the extent of the tumor, and the frozen sections of biopsy revealed SCC of the larynx. The postoperative period was uneventful and recovery was uneventful.

Postoperative histopathological examination revealed SCC grade II, with the tumor extending to the cartilage, all the cartilages free, and the tracheal cut margins free. Preepiglottic and paraglottic space involvement was present. Three nodes in level 2a had metastatic spread without extracapsular spread, while all the left level $2 b$, level 3 , level 4 nodes had no metastasis. All the right-sided nodes were reactive. L eft sided hemithyroidectomy specimen was free of tumor. The patient had postoperative concurrent chemoradi otherapy with 4 cycles of radical CDDP weekly with external beam radiotherapy of 54 grays. The patient is being followed up with 18 months of disease free survival. The patient is trained for electrolarynx as the patient could not afford the costs of voice prosthesis and second surgical intervention of trachea esophageal puncture (Fig. 2).

\section{DISCUSSION}

L aryngeal cancers are malignancies of old age, common in males and usually present in the 5 and 6 th decade of life. ${ }^{3}$

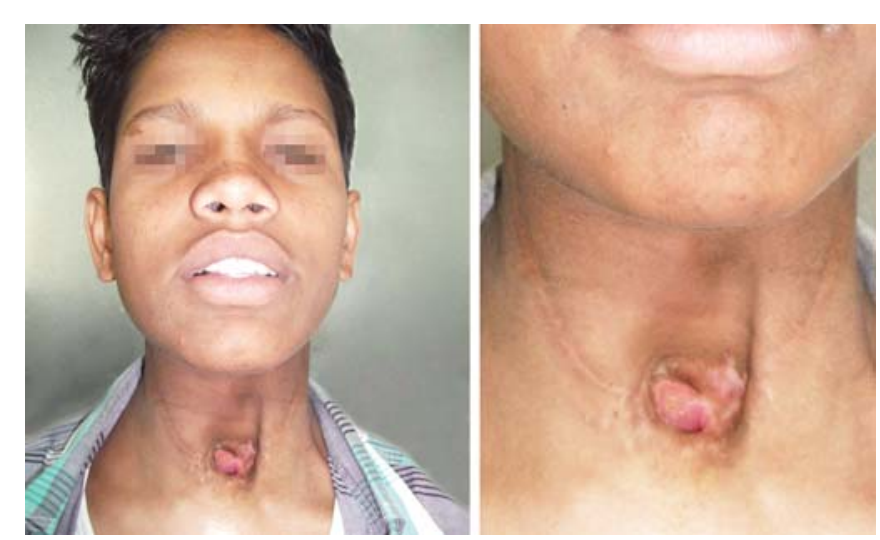

Fig. 2: Post wide field laryngectomy and radiotherapy stoma

Laryngeal papillomatosis is the commonest laryngeal neoplasm in children and supraglottic carcinoma is common laryngeal carcinoma among the literature reported so far. $^{3}$

$M$ anish et al in literature search on 71 cases found increased female incidences, $10 \%$ in 0 to 5 years, $23 \%$ in 5 to 10 years and $63 \%$ in 10 to 15 years age group. ${ }^{5}$ The lesions were common in glottis $78 \%$, while in supraglottis was $17 \%$ and $5 \%$ in subglottis. ${ }^{5,14}$ Early and advanced cases were equally seen in these laryngeal subsites. ${ }^{5} \mathrm{M}$ ost of the cases were SCC while adenocarcinoma, mucoepidermoid carcinoma, adenoid cystic carcinoma and clear cell carcinoma, metastasis to larynx were al so reported. ${ }^{15-18} \mathrm{SCC}$ with neck nodes metastasis have been reported in one case. ${ }^{15-18} \mathrm{All}$ the patients had prolonged symptoms of hoarseness or upper airway obstruction and often overlooked as voice changes during puberty, recurrent upper respiratory tract infection (URTI) or vocal abuse. ${ }^{15-18}$

Difficulty in eliciting history examination, clinical and imaging studies added to the woes of the surgeon. ${ }^{5}$ M ost common predisposing factor in most of the cases were irradiation of benign lesions of the head and neck, especially juvenile laryngeal papilloma. ${ }^{8,19,20}$ As the condition resembles benign lesions and possibility of it included as differential diagnosis is rare and difficulty in clinical examination due to recurrent respiratory tract infection, or asthma, vocal abuse makes the disease more advanced at presentation. ${ }^{9,10}$

Etiologies included are irradiation for recurrent respiratory papillomatosis and HPV infection. ${ }^{11,21}$ Spontaneous malignant changes in the recurrent respiratory papillomatosis is also reported in the laryngotracheal and bronchoalveolar regions with an incidence rate of $2.3 \%{ }^{22}$

Stephen et al reported HPV 16, is a causative agent for $25 \%$ of head and neck SCC, including laryngeal squamous cell cancer (LSCC). ${ }^{23}$ They reported no correlation of better survival of HPV-positive oropharyngeal tumors when compared to laryngeal lesions. ${ }^{23}$ First, Zahidul et al reported 
that H PV 11 had been linked to the malignant transformation of juvenile-onset recurrent respiratory papillomatosus. ${ }^{24}$ $M$ alignant transformations of papillomatosis were $14 \%$ ( 6 of 43 irradiated cases) below 30 whereas no transformation was reported in 58 cases treated with surgery al one..$^{25}$

Torrente et al reported detection of HPV DNA in benign (papillomatosis), indolent (verrucous carcinoma) and malignant (SCC) lesions of the larynx.$^{26} \mathrm{HPV}$ types associated with laryngeal papillomatosis include low-risk HPV types 6 and 11, with high-risk HPV types 16 and 18 more commonly present in neoplastic lesions (verrucous carcinoma and SCC). ${ }^{26} \mathrm{~A}$ total of $25 \%$ of laryngeal SCCS harbor HPV infections on meta-analysis, with common involvement of high-risk HPV types 16 (highest frequency) and $18 .{ }^{26} \mathrm{High}$-risk H PV infections seem to be biologically relevant in laryngeal carcinogenesis, manifested as having viral DNA integration in the cancer cell genome and increased expression of the p16 protein. ${ }^{26}$ The clinical significance of these infections and the implications on disease management are unclear and require active research. ${ }^{26}$

With the analysis of the nearly 100 cases in literature a working guidelines has been drawn where supraglottic lesions behaves aggressively and so should be treated radically. ${ }^{5}$ Laryngeal preservation can be thought of in glottis lesions as they have a favorable outcome. ${ }^{5}$ The treatment options are individualized according to the institutional policies and facilities available as no evidencebased management guidelines are available. ${ }^{5}$ Commonly surgery have been the main treatment protocol, while the role of radiotherapy and chemotherapy are not yet defined. ${ }^{5}$ Radiotherapy can cause significant growth retardation of both soft tissue and bone with consequent deformity and dysfunction and long-term complications like facial grow th retardation, neuroendocrine dysfunction, visual problems, dental abnormalities and hypothyroidism. ${ }^{5}$ L ocoregionally late side effects after a decade after radiotherapy include chondronecrosis, esophageal stenosis, second malignancy and brain hemorrhage. ${ }^{27}$

Gindhart et al reviewed 17 cases of childhood laryngeal SCC and found that seven had fatal course before definitive treatment, 10 cases operated, or with radiotherapy or postoperative radiotherapy had disease free survival of 20 months. ${ }^{1}$ They reviewed 13 glottic lesions where stripping was done in two, partial laryngectomy in three, total laryngectomy in two, radiotherapy in three, total laryngectomy plus radiotherapy in one and chemotherapy plus radiotherapy in two. ${ }^{2,28}$ Though long-term follow-up is not reported for all these cases, local recurrence was seen in only two patients, both of which were successfully salvaged. ${ }^{1,29}$ The four cases of supraglottic primaries, two died from rapidly progressive disease and two were irradiated. 6,30,31

Mayo clinic in their study on 101 cases of laryngeal papilloma reported six of the 43 cases (14\%) irradiated and devel oped SCC before 30 years, while the rest operated did not show malignant degeneration. ${ }^{25}$ Other etiologies include infection with HPV 18 and 33, active and passive smoking and exposure to chemical agents like asbestos and a rare chromosomal translocation 15:19 associated with supraglottic tumors. ${ }^{4}$ Patients in the age group 12 to 17 years with laryngeal cancers have tobacco smoking and chewing as principal risk factors. ${ }^{9}$

\section{CONCLUSION}

Pediatric laryngeal cancers are very rare and studies about the etiology, biology of the tumor, diagnostic and management protocols are not standardized. Diagnosis should be thought of in cases with hoarseness of long duration, cough or upper airway disease not being controlled with aggressive medical treatment and associated with risk factors. Formulating a protocol for childhood laryngeal cancers management which includes early diagnosis and accurate treatment is essential.

\section{REFERENCES}

1. Gindhart TD, J ohnston WH, Chism SE, et al. Carcinoma of the larynx in childhood. Cancer 1980;46:1683-87.

2. O hlms LA, McGill T, Healy GB. Malignant laryngeal tumors in children: A 15-year experience with four patients. A nn Otol Rhinol Laryngol 1994;103:686.

3. Islam ZM , A hmad SM , Huda M M. Carcinoma larynx in a 10year-old girl: A rare clinical entity. Bangladesh J Otorhinolaryngol 2012;18(1):68-73.

4. Simon M, K ahn T, Schneider A, Pirsig W . L aryngeal carcinoma in a 12-year-old child. A ssociation with human papilloma virus 18 and 33. A rch O tolaryngol Head N eck Surg 1994;120:277-82.

5. J ulaha $M$, Chaturvedi $P$, Pai $P$, Chaukar D, Pantvaidya Gauri D'C ruz A . Carcinoma larynx in children. IntJ Head N eck Surg 2010 J an-A pr 2010;1(1):49-51.

6. R astogi M, Srivastava M , B hatt M L B, et al. L aryngeal carcinoma in a 13-year-old child. Oral Oncol Extra 2005;41:207-10.

7. J ones AC. Carcinoma of the larynx in a girl aged 15 . A rch 0 tol 1942;35:426-30.

8. M cGuirt WF, Little JP. Laryngeal cancer in children and adolescents. Otolaryngol Clin North A m 1997;30:207-14.

9. Faragó L, Nagy A. A rare case of malignant degeneration of a childhood laryngeal papilloma. Mschr Ohrenheilk 1966;100:131-35.

10. Rutt $A L$, Hawkshaw MJ, Sataloff RT. Laryngeal cancer in patients younger than 30 years: A review of 99 cases. Ear Nose Throat J 2010;89(4):189-92.

11. Ossoff RH, Tucker GF, Norris CM . Carcinoma of the larynx in an 11-year-old boy with late cervical metastasis: Report of a 
Pediatric Laryngeal Malignancies: Current Management P rotocols and Review of Literature

case with 10-year follow-up. Otolaryngol Head Neck Surg 1980;88:142-45.

12. de Carval ho M B , et al. Head and neck squamous cell carcinoma in childhood. M ed Pediat Oncol 1998;31:96-99.

13. Birchall MA, Pope L. Tumours of the larynx. Scott-Brown's Otorhinolaryngology: Head and neck surgery (7th ed). In: Gleeson M , A rnold E (Eds) 2008;194:2598-622.

14. Gaylis B, Hayden RE. Recurrent respiratory papillomatosis: Progression to invasion and malignancy. A m J Otolaryngol 1991;12:104-12.

15. Haghighi P, M ohallatee FA, N as K, Dezhbakhsh F, Salmasi S, et al. Childhood cancer in Southern Iran. Cancer 1974;34: 1842-48.

16. M itchell DB, Humphreys S, K earns DB. M ucoepidermoid carcinoma of the larynx in a child. IntJ Pediatr Otorhinolaryngol 1988;15(2):211-15.

17. Javadi M, B afrouee FM, M ohseni M, A sghari A. Laryngeal adenoid cystic carcinoma in a child: A case report. Ear Nose Throat J 2002;81(1):34-35.

18. Nayak DR, Balakrishnan R, Rao RV, Hazarika P. Clear cell carcinoma of the larynx: A case report. Int J Pediatr Otorhinolaryngol 2001;57(2):149-53.

19. Rabbett WF. J uvenile laryngeal papillomatosis: The relation of irradiation to malignant degeneration in this disease. A nn Otol Rhinol Laryngol 1965;74:1149-63.

20. Walsh TE, B eamer PB. Epidermoid carcinoma of the larynx occurring in two children with papilloma of the larynx. Laryngoscope 1960;60:1110-24.

21. Walkinson JC, Gaze M N, W ilson JA. Tumours of the larynx. Stell and M aran's, Head and Neck Surgery (4th ed). Oxford: Butterworth Heinemann 2000;15:233-73.

22. M oore CE, W iatrak BJ, M CClathey K D, K oopman CF, Thomas $G R, B$ radford CR, Carey TE. High-risk human papillomavirus types and squamous cell carcinoma in patients with respiratory papilloma. Otolaryngol H ead N eck Surg 1999;120(5):698-705.

23. Stephen JK, Chen KM, Shah V, Havard S, Lu M, Schweitzer V P, Gardner G, W orsham M J. H uman papillomavirus outcomes in an access-to-care laryngeal cancer cohort. O tolaryngol Head Neck Surg 2012 M ay;146(5):730-38.

24. French $C$, Kutok J, Faquin W, et al. Midline carcinoma of children and young adults with NUT rearrangement. J Clin Oncol 2004;22:4135-39.

25. M ajoros M , D evine K D, Parkhill EM . M alignant transformation of benign laryngeal papilloma in children after radiation therapy. Surg Clin North A m 1963;43:1049-61.

26. Torrente M C, Rodrigo J P, Haigentz M J r, Dikkers FG, Rinaldo $A, T$ akes RP, et al. Human papillomavirus infections in laryngeal cancer. Head N eck 2011 A pr;33(4):581-86.
27. Paulino AC, Simon JH, Zhen W, W en B. L ong-term effects in children treated with radiotherapy for head and neck rhabdomyosarcoma. Int J Radiat Oncol Biol Phys 2000;48: 1489-95.

28. Laurian N, Sadov R, Strauss M , K essler E. L aryngeal carcinoma in childhood. Report of a case and review of the literature. L aryngoscope 1984;94:684-87.

29. B arnes C, Sexton M , Sizeland A, Tiedemann K, B erkowitz R G, Waters K. Laryngo-pharyngeal carcinoma in childhood. Int J Pediatr Otorhinolaryngol 2001;61:83-86.

30. Siddiqui F, Sarin R, A garwal JP, Thotathil Z, M istry R, et al. Squamous carcinoma of the larynx and hypopharynx in children: A distinct clinical entity? M ed Pediatr Oncol 2003;40:322-24.

31. Goyal P, K ellman R M. Frontolateral hemilaryngectomy for the management of a case of pediatric squamous cell carcinoma of the larynx. L aryngoscope 2005;115:965-67.

\section{ABOUT THE AUTHORS}

\section{Sudhir M Naik (Corresponding Author)}

Fellow, Department of Head and Neck Oncosurgery, Kidwai M emorial InstituteofOncology,Bengaluru,K arnataka,India,e-mail:sud223@ gmail.com

\section{A Nanjundappa}

Professor, Department of Head and Neck Oncosurgery, Kidwai M emorial Institute of Oncology, B engaluru, K arnataka, India

\section{H Srihariprasad}

Resident M edical Officer, Department of H ead and Neck Oncosurgery Kidwai M emorial Institute of Oncology, Bengal uru, K arnataka, India

\section{Rajshekar Halkud}

A sociate Professor, Department of Head and Neck Oncosurgery Kidwai M emorial Institute of Oncology, Bengal uru, K arnataka, India

\section{Purshottam Chavan}

Assistant Professor, Department of Head and Neck Oncosurgery Kidwai M emorial Institute of Oncology, Bengaluru, K arnataka, India

\section{Sumit Gupta}

Fellow, Department of Head and N eck Oncosurgery, K idwai M emorial Institute of Oncology, B engaluru, Karnataka, India 